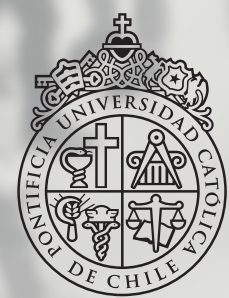

PONTIFICIA

UNIVERSIDAD

CATÓLICA

DE CHILE
Revista

Trabajo
Análisis crítico

de la teoría de cambio en intervenciones de visitas domiciliarias

en el programa

Chile Crece Contigo

Kenichi Haramoto

Paula Verdugo

Visita domiciliaria en Trabajo Social:

¿cómo la valoran y utilizan un grupo de estudiantes de la profesión?

Paula Chamorro

Alicia Razeto
Propuesta de un modelo de supervisión reflexiva de visitas domiciliarias realizadas en un programa dirigido a familias de recién nacidos

María Carolina Velasco Hodgson

Daniela Tuma IBIETA

Claudia Aldana Pereira

\section{Formación continua en} visita domiciliaria.

Análisis cualitativo de una metasupervisión reflexiva

Claudia Cerfogli

Paulina Contreras

Experiencias sobre el uso de eHealth en educación para la salud, gestación y primera infancia:

una revisión bibliográfica

Benjamin Charles Nielsen Guzmán 



\title{
Formación continua en visita domiciliaria. Análisis cualitativo de una metasupervisión reflexiva
}

\section{Continuous training in home visit. Qualitative analysis of a reflexive metasupervision}

\author{
Claudia Cerfogli \\ Magíster en Psicología Clínica, UC. ccerfogl@uc.cl \\ Paulina Contreras \\ Magíster en Psicología de la Salud, UC. pacontrerasm@uc.cl Pontificia Universidad Católica de Chile, Escuela de Psicología. Av. \\ Vicuña Mackenna 4860, Macul.
}

\begin{abstract}
Resumen
El rol de la monitora de visita domiciliaria en primera infancia se ha ido visibilizando en el último tiempo y ha emergido la importancia de la relación en esa visita, por sobre la entrega de contenidos o educación. Emerge así la necesidad de trabajar con la persona del agente visitador, más allá de la necesaria capacitación, siendo necesario generar un programa que integre tanto una capacitación constante como un apoyo mantenido en el tiempo al agente visitador.

En el marco del proyecto Fondecyt 1100762 sobre Visita Domiciliaria en Salud, se genera un programa piloto de visita domiciliaria que incluye capacitación, supervisión y metasupervisión que permiten garantizar distintos niveles de apoyo y capacitación a las monitoras. El presente trabajo se enfoca en el análisis particular de esa metasupervisión.

Se realiza un estudio cualitativo, utilizando la Grounded Theory, analizando las sesiones de metasupervisión y dos entrevistas individuales a supervisora y metasupervisora. Desde el análisis se confirman aspectos de los modelos teóricos revisados, como la presencia en la metasupervisión de aspectos característicos de la supervisión reflexiva, como son la reflexión, la colaboración y la regularidad. Aparecen temas emergentes en relación con la satisfacción y desarrollo profesional del supervisado, influidos por la metasupervisión. Finalmente, se realiza una reflexión crítica sobre los alcances del presente estudio.
\end{abstract}

Palabras claves: Supervisión reflexiva, metasupervisión, supervisora, maternidad, metodología cualitativa.

\footnotetext{
Abstract

The role of the home visitation monitor in early childhood has been visible, and has emerged the importance of the relationship in that visit over the delivery of content or education. Thus highlights the need to work with the person of the visiting agent, beyond the necessary training, being necessary to generate a program that integrates both a constant training and a support maintained over time to the visiting agent.

In the framework of the Fondecyt project 1100762 on Home Health Visits, a pilot program in home visit is generated that includes training, supervision and metasupervision that allow different levels of support and training to be guaranteed to the monitors. The present work focuses on the particular analysis of that metasupervision.

A qualitative study is carried out using the Grounded Theory, sessions of metasupervision and two interviews, one with supervisor and one with metasupervisor are analized. From the analysis aspects of the revised theoretical models are confirmed, such as presence in metasupervision of aspects of reflexive supervision, such as reflection, collaboration and regularity. Emerging issues appear in relation to professional satisfaction and development of the supervised, influenced by the metasupervision. Finally, a critical reflection on the scope of the present study are made.
}

Keywords: Reflexive supervision, metasupervision, supervisor, maternity, qualitative methodology. 


\section{Introducción}

El rol de la monitora de visita domiciliaria a primera infancia se ha ido visibilizando y ha emergido la importancia de la relación con la madre o familia en esa visita por sobre la entrega de contenidos o educación. Emerge así la necesidad de trabajar con la persona del agente visitador, más allá de la necesaria capacitación, siendo necesario generar un programa que integre tanto una capacitación constante como un apoyo mantenido en el tiempo al agente visitador. En el marco de un proyecto Fondecyt (1100762 sobre Visita Domiciliaria en Salud), se genera un programa piloto de visita domiciliaria que incluye capacitación, supervisión y metasupervisión que permiten garantizar distintos niveles de apoyo y capacitación a las monitoras. El presente trabajo se enfoca en el análisis particular de esa metasupervisión.

La labor del supervisor de monitores es esencial, y a su vez este necesita apoyo para su labor, encarnado en un metasupervisor que orienta y le permite mantenerse articulado a los objetivos macros de las políticas públicas donde se han insertado.

El presente estudio busca reconocer las creencias de parte del supervisor sobre su modelo de trabajo, las temáticas más recurrentes que son objeto de supervisión, la interacción que surge dentro de la díada de supervisión y, junto con esto, las percepciones de la supervisada y metasupervisor sobre el fenómeno observado. Se busca develar la relación entre estos fenómenos para poder dar cuenta de aspectos característicos de una metasupervisión.

A través de un estudio de caso único, se realizó un seguimiento durante diez meses de sesiones mensuales de metasupervisión. Se transcribieron los audios y se analizaron cualitativamente con datos emergentes y con la búsqueda de categorización de los mismos. Además, se realizó una entrevista en profundidad a la supervisora para averiguar sus creencias y percepciones sobre el quehacer de supervisión; entrevista que fue transcrita y analizada cualitativamente. Al finalizar el proceso de supervisión, se realizó una entrevista semiestructurada a la supervisada, que permitió conocer la percepción de la misma sobre el proceso de supervisión y su grado de satisfacción en torno a él. Esta entrevista fue transcrita y analizada cualitativamente.

Mediante estas entrevistas, se buscó poder observar cambios y permanencias dentro de la relación de supervisión de parte de ambos participantes. Esos cambios pueden observarse en el análisis en el tiempo. A partir de lo anterior se pretende dar luces sobre las necesidades de supervisión, el perfil del supervisor, el modelo utilizado para este proceso que permita diseñar el plan de acción de las políticas públicas sobre la asistencia a monitores.

Considerando lo anterior, mediante este análisis la pregunta que se espera responder es: ¿Cuáles son los aspectos que caracterizan una metasupervisión en el contexto de las visitas domiciliarias en salud en primera infancia?

Dentro del programa de visita domiciliaria se incluye la participación de la supervisora de monitoras en una metasupervisión guiada por un profesional psicólogo clínico. Esta instancia se concibe en el marco de una supervisión reflexiva donde el centro de intervención es la persona misma del supervisado y la relación con su supervisor. En el artículo desarrollado en esta misma publicación, se despliega ampliamente el modelo concebido desde el cual emerge esta instancia de metasupervisión (Velasco, Tuma, Aldana, 2015). Brevemente, podemos señalar la importancia en esta supervisión de enfocarse en las necesidades del supervisado para poder mejorar su desempeño en el rol, pero incluyendo las posibilidades de generar un espacio que trasciende la tarea y apoya al supervisor en un amplio rango de necesidades (respecto de su propio desarrollo personal, profesional y satisfacción). Junto con lo anterior, surge un paradigma que da cuenta de mirar la supervisión como un proceso de encuentro y coconstrucción de realidades donde la relación entre metasupervisor y supervisado es lo que media para que ese proceso sea suficientemente significativo para mejorar la percepción del propio desempeño en el rol.

Los procesos de coconstrucción en los fenómenos de cambio llevan a concebir este último como posible desde la capacidad que se tiene para aceptar la mirada del otro como válida y necesaria en el camino de las mejoras de las propias prácticas. El encuentro con otro permite enriquecer la mirada muchas veces rigidizada y saturada de problemas del supervisor. Este estudio de la metasupervisión permite visibilizar este encuentro para relevar su importancia dentro de un programa de acompañamiento en salud mental.

El compromiso con los niños dentro de la sociedad es cada vez más fuerte. Tenemos evidencia de la importancia de los primeros años en el desarrollo ulterior de la persona, consecuencias, algunas veces determinantes para lograr la plenitud personal en distintos ámbitos. Hoy sabemos que el aprendizaje comienza desde el período gestacional y que depende, centralmente, de la relación con sus figuras significativas (Fonagy y Target, 2002; Gunnar, Mangelsdorf, Larson y Hertsgaard, 1989). 
Los profesionales en contacto con la primera infancia saben que el desarrollo del cerebro se despliega a través de la crianza, y los bebés nacen con muchas capacidades que los predisponen a un aprendizaje desde lo relacional, incluyendo la memoria, la imitación y la interacción (Greenspan, 1992; Shonkoff y Phillips, 2000). Lo anterior lleva a que los distintos agentes sociales estén más alertas y comprometidos con generar en ellos destrezas que les permitan facilitar este encuentro entre los padres y sus hijos desde los primeros momentos de vida.

A su vez, los profesionales que trabajan con los padres y los niños necesitan sentirse apoyados en su trabajo para acompañar adecuadamente a una familia; esto se conoce como el proceso paralelo (Geller, Faber y Shaffer 2010; Weatherston y Barron, 2009). La premisa del proceso paralelo es que las relaciones se van ampliando; lo que ocurre en una relación puede inmensamente impactar otras relaciones (Geller, Whiteman y Rosenthal, 2010). La supervisión reflexiva está basada en el proceso paralelo. Esto significa que, para cuidar bien de una familia, debemos cuidar de toda la cadena de profesionales en relación con ella (Ruhe Mach, 2012) Gill y colegas (Gill, Greenberg, La Luna y Margraf, 2007) documentan altos niveles de agotamiento emocional y depresión en personal de visitas domiciliarias haciendo necesaria una mayor nivel de supervisión y apoyo (Jones Harden, 2010).

La supervisión reflexiva se ha definido como el "proceso de examinar con otra persona los pensamientos, sentimientos, acciones y reacciones evocadas en el curso del trabajo con los jóvenes y sus familias" (Eggbeer, Main y Seibel, 2007, p. 5). La supervisión reflexiva se ha convertido en un elemento esencial de los programas de visitas domiciliarias (Eggbeer et al., 2007; Heffron, 2005). Se entiende en estos programas que la visita domiciliaria de los distintos profesionales o monitores no debe ser una práctica aislada sino que en colaboración con un supervisor de apoyo; este proceso se conoce como la supervisión reflexiva (Kraemer, 2006). En el contexto de una relación de confianza, por lo general con el supervisor del programa, el visitador habla de sus sentimientos, ideas, observaciones y los sentimientos de las visitas domiciliarias previas (Weatherston, Kaplan- Estrin, y Goldberg, 2009).

Al participar en este proceso, los visitadores son apoyados y escuchados y favorece la actitud más positiva en su trabajo con niños y familias (Weatherston y Barron, 2009). La naturaleza emocional del trabajo con familias en situaciones de vulnerabilidad puede resultar altamente agotador y desafiante favoreciendo la alta rotación de los visitadores en los programas (Wasik \& Richard, 2004; Weld, 2011). Desde ahí, el objetivo de la supervisión reflexiva es permitir que quien realiza la visita domiciliaria tenga un ambiente seguro para mirar su trabajo, resolver problemas y rescatar las capacidades, las vulnerabilidades y las experiencias de vida que pueden afectar las intervenciones en las familias (Gilkerson, 2004).

A su vez, los supervisores de los monitores también requieren de un espacio de reflexión sobre sus prácticas de supervisión. Este espacio se materializa en una metasupervisión que permite completar la cadena de apoyo y sostén del programa, favoreciendo la mirada flexible y dinámica del proceso de acompañamiento a las madres y sus hijos.

Tanto la supervisión como la metasupervisión se construyen en el paradigma de una supervisión reflexiva. En ésta se ha podido determinar algunos elementos característicos que son la reflexión, la colaboración y la regularidad (Parlakian, 2001).

Por reflexión nos referimos a tomar distancia y darse el tiempo para pensar sobre lo que la experiencia realmente significa. Incluye examinar pensamientos, sentimientos sobre la experiencia y mirar las propias acciones de un modo crítico para poder desarrollar las más eficientes para el desarrollo de esa familia y del profesional a cargo también.

Esta reflexión se basa en una relación de confianza y honestidad para que pueda darse esta exploración. Aquí el papel del supervisor será facilitar esa exploración en el supervisado y dar los apoyos y los conocimientos necesarios para orientar la toma de decisiones. Incluye también la posibilidad de que el supervisor favorezca en el supervisado un andamiaje para su propio desarrollo profesional, más allá del trabajo que lo convoca a esa supervisión. El espacio de supervisión pasa a ser un espacio de autoconocimiento de las propias fortalezas, los límites y dificultades. Este proceso permite la mejora progresiva en el desempeño laboral.

Por colaboración se entiende el compartir la responsabilidad del accionar social dentro de un equipo. Quien realiza la visita domiciliaria percibe que comparte esa responsabilidad con un equipo cuya cara visible es el supervisor. A su vez, en una metasupervisión, el supervisor de las visitas tiene la posibilidad de compartir esa responsabilidad con su metasupervisor. El ambiente de colaboración requiere de un clima de confianza e intimidad que favorezca una mirada ajena al juicio de valor, donde el metasupervisor debe caracterizarse por una legítima curiosidad, escucha activa y receptividad. 
Por último, al hablar de regularidad nos referimos a que una supervisión debe darse en un terreno plausible y previsible, debe haber una regularidad en las interacciones tanto en tiempo como en espacio evitando las cancelaciones y reprogramaciones que, igualmente, se dan en los contextos de trabajo comunitario. Brenda Jones Harden (2010) lo releva como un aspecto fundamental dada la especial sensibilidad que implica el trabajo directo con familias en riesgo psicosocial.

Se puede determinar que existen ciertas competencias en el supervisor que se requieren para poder llevar a cabo la relación fructífera hacia procesos de reflexión y desarrollo profesional y personal. Estas competencias son tanto fundacionales, comunes a todo profesional, como específicas del rol de supervisor de agentes en visita domiciliaria. Las primeras son: habilidades interpersonales, conocimiento de lo ético, legal y políticas públicas, capacidad reflexiva de autoevaluación, conocimientos técnicos, capacidad de trabajo en equipo, entre otras (Rodolf Rodolfa, E., Bent, R., Eisman, E., Nelson, P., Rehm, L. Ritchie, P. 2005; Morgan y Sprenkle, 2007). Las profesionales se refieren al ejercicio específico de la disciplina supervisada.

En el caso de la metasupervisión en visita domiciliaria, tenemos la capacidad de conocer sobre distintos ámbitos, desde el desarrollo en primera infancia, maternidad, trabajo en visita domiciliaria a requerimientos de un programa institucional, objetivos del mismo, etc. Requiere ser experto en procesos de formación de profesionales, en temas administrativos y de gestión en que estos están involucrados. Chang (2013) realiza un metaanálisis sobre los elementos que conformarían los roles de un supervisor, entre los cuales se cuenta con que tengan en cuenta el contexto administrativo, generen una relación de alianza con el supervisado, tomen en cuenta los distintos roles del supervisado y las distintas fases en que el supervisado se encuentra, tanto del ejercicio mismo como de su experticia profesional y momento vital. Es importante resaltar que el autor también considera un rol esencial del supervisor el ser garante de la mejora continua de los procesos de ayuda hacia la comunidad, equilibrando las necesidades del supervisor con los objetivos del programa y con las necesidades de las familias a las que se atiende.

\section{Metodología}

Diseño. El presente estudio se inserta dentro de un programa piloto de visitas domiciliarias a madres gestantes y con hijos en sus primeros meses de na- cido. Este programa es un proyecto de investigación de la Escuela de Psicología de la Universidad Católica de Chile, que consiste en una intervención a través de visitas domiciliarias a madres de recién nacidos pertenecientes a un centro de salud familiar durante un año. Las monitoras seleccionadas para las visitas corresponden a paraprofesionales de la comunidad.

Para la presente investigación exploratoria se utilizó un diseño descriptivo, relacional y analítico, empleándose la metodología cualitativa (Flick, 2004), donde el análisis de resultados se sustentó en el modelo de la Grounded Theory, desarrollado por Glaser y Strauss (1967). La perspectiva cualitativa resultó especialmente pertinente en esta investigación, ya que permitió acceder a los procesos y significados subjetivos de quienes participan del proceso de metasupervisión (Ruiz, 1996; Flick, 2004). A su vez, la Grounded Theory constituye un método privilegiado para realizar este proceso, justamente porque busca construir modelos teóricos acerca de las interacciones de los diferentes aspectos del fenómeno en estudio (Krause, 1998), por medio de procesos inductivos y holistas (Taylor \& Bodgan, 1986); resultó pertinente para el desarrollo de conceptos bien integrados, otorgando una explicación teórica detallada y precisa de los fenómenos sociales estudiados (Glaser \& Strauss, 1967; Strauss \& Corbin, 1990). Este enfoque permite tanto el estudio de las características del programa en cuanto a sus contenidos, y dinámicas de quienes participan en la supervisión y las características propias que se dan en este tipo de relación.

Participantes. El programa de visitas en que se da este estudio contempla la visita a madres desde la gestación hasta los primeros meses de vida del bebé, de parte de dos monitoras. Estas monitoras son supervisadas por una supervisora, psicóloga, quien es parte del presente estudio. Finalmente, esta supervisora asiste a metasupervisión mensual con una metasupervisora, psicóloga clínica, experta en supervisión (supervisora acreditada por la Sociedad Chilena de Psicología Clínica).

En esta investigación se realizó un muestreo con un grupo definido de antemano (Flick, 2004), que corresponde a la supervisora de las monitoras y la supervisora de ésta última, denominada en este trabajo como metasupervisora, y al espacio de trabajo de ambas como metasupervisión.

Procedimiento. Se tomaron los audios de las sesiones de metasupervisión mensuales entre los meses de abril a diciembre de 2014, con un total de 8 audios de supervisiones, ya que en julio no 
se realizó supervisión. Estas fueron transcritas y analizadas. Adicionalmente se realizaron entrevistas individuales con cada integrante supervisora y metasupervisora, por separado. Las entrevistas se realizaron en dependencias de la universidad con una duración aproximada de una hora, para lo cual se desarrolló un guion de entrevista, cuyos ejes principales fueron: contenidos de la metasupervisión, obstaculizadores/facilitadores de la metasupervisión, paradigmas de metasupervisión y relación de la díada en ese espacio.

Estrategias de producción de datos. La producción de información se realizó por un lado mediante la transcripción de los audios de supervisión mensual. Estas sesiones de supervisión habían sido registradas por sistema de audio, a través de una grabadora que fue manipulada por la supervisada, mientras que las entrevistas se condujeron con un guion temático y la grabación fue manipulada por la entrevistadora (Taylor \& Bogan, 1986).

Análisis de datos. La codificación de los datos se llevó a cabo a través de tres etapas o procesos: Codificación abierta, axial y selectiva, como parte del modelo de Grounded Theory. En la primera de ellas se realizó un proceso de examen, fragmentación y comparación de la información obtenida, con el fin de reagruparla de acuerdo a la elaboración de categorías, construida mediante la identificación de fenómenos relevantes, los cuales son descritos a través de propiedades y dimensiones. La segunda correspondió a una nueva forma de codificación mediante la conexión entre categorías, utilizando un paradigma de análisis que involucra al fenómeno, su contexto general de aparición, las condiciones causales intervinientes, estrategias de acción o interacción y finalmente sus consecuencias. Y la tercera correspondió a la codificación selectiva, involucrando la selección de una categoría central, en torno a la cual los datos fueron sometidos a una comparación permanente, que permitió extraer los significados de la experiencia reiterativos del discurso (Strauss \& Corbin, 2002). Finalmente, el tipo de resultados que se obtuvo fueron descriptivos y analítico-relacionales, dado el tipo de codificación realizada durante el análisis de los datos recopilados durante el desarrollo de la investigación.

La triangulación de investigadores y de perspectivas teóricas fueron los criterios de rigurosidad y calidad que se emplearon en la presente investigación y que le entregó rigor científico mediante el juicio de credibilidad (Pedersen, 1992).

Consideraciones éticas. Este estudio protegió los derechos, privacidad y bienestar de las personas involucradas. Se les entregó información respecto a sus objetivos, de forma clara y en un lenguaje adecuado para los usuarios. Asimismo, el proceso investigativo estuvo a cargo de profesionales responsables y respaldados por la Pontificia Universidad Católica de Chile.

Se consideró la inexistencia de algún riesgo potencial para los participantes, quienes fueron respetados en todo el proceso investigativo y tuvieron libertad para participar o retirarse durante el proceso. Se garantizó la confidencialidad de su identidad, generándose un consentimiento por parte de los participantes. Se utilizó el protocolo APA y la declaración de Helsinki para el resguardo de este punto.

Para garantizar el resguardo de la información y la identidad de las participantes, sus nombres fueron omitidos, conservando su anonimato. En este sentido, fueron pilares éticos fundamentales la confidencialidad, la privacidad, el fomento de la participación, el respeto por la autonomía y la autodeterminación de los participantes.

\section{Objetivos}

\section{Objetivo General:}

Describir y analizar un proceso de metasupervisión de monitoras de visitas domiciliarias en salud.

\section{Objetivos Específicos:}

- Caracterizar los principales elementos de la metasupervisión.

- Identificar los principales contenidos de la metasupervisión.

- Identificar y describir las características del metasupervisor.

- Identificar la percepción de las participantes respecto de la metasupervisión.

\section{Resultados descriptivos}

\section{Temas principales}

En el espacio de metasupervisión se observa, frecuentemente, que es la supervisada (S) quien trae los temas al espacio de supervisión. Uno de esos temas recurrentes es la relación con las monitoras en la supervisión individual y grupal que realiza con ellas. En este sentido, aparecen contenidos en relación con los diferentes estilos de las monitoras, al manejo de la visita domiciliaria por parte de ellas y a las expectativas asociadas a su rol, entre otros. Otros temas recurrentes de este espacio son la explicitación de lo que es una visita domiciliaria desde el modelo del programa (centrada en el vínculo con la madre versus sólo la entrega de información 
de salud), el rol de la psicóloga del centro de salud dentro de las supervisiones con las monitoras, madres en distintos momentos del puerperio o con problemas de riesgo de consumo de sustancias o violencia intrafamiliar.

Otro tema importante de la supervisión es el rol múltiple que despliega $S$ con respecto al programa y a las monitoras, el cual influye en la relación y satisfacción de ésta con las monitoras; por ejemplo, aparece que ella, además de supervisar semanalmente a las monitoras, es quien coordina el pago del sueldo y debe resolver los problemas asociados (retraso en el ingreso del dinero, etc.). Se observan algunos cambios en las temáticas a ser supervisadas de las cuales daremos cuenta en el párrafo siguiente. Hacia el final de las supervisiones analizadas se observan otros temas relacionados con la satisfacción y proyección del rol en la monitora y la supervisada, y temas de cierre del proceso de visita domiciliaria con las madres.

\section{Cambios en los contenidos}

Se incorpora en un comienzo temáticas de dificultad a la metasupervisión que requieren una resolución rápida (por ejemplo, estilos de enfrentamiento de las monitoras, la relación de éstas con las madres o con ella, las inasistencias de otro miembro del equipo en la supervisión con las monitoras en forma reiterada). Luego, se incorporan contenidos como la multiplicidad de roles de S, temáticas de violencia de pareja y de abuso de sustancias de algunas de las madres. Estos temas se mantienen a lo largo de la supervisión; sin embargo, se observa la inclusión posterior de otros contenidos que van más allá de las dificultades, como los logros de las monitoras en su experiencia personal y en su actividad como monitoras con las madres, y la potenciación de sus recursos, siendo reconocidas en otros ámbitos (por ejemplo, al invitarlas a la capacitación de médicos familiares). Este ejemplo puede estar dando cuenta de una ampliación en el significado de la metasupervisión, en el que se van incluyendo otras temáticas, dando un espacio para relevar los recursos y habilidades personales y profesionales de los miembros involucrados. Hay un énfasis en los recursos y en las capacidades de los integrantes: "lo otro es que no sé si te había contado, que la psicóloga... (Refiriéndose a la profesional del centro de salud) hace algunas clases de medicina familiar y las invito a ellas (las monitoras) para que contaran su experiencia de monitoras comunitarias en salud a médicos que se están formado como médicos familiares" $(S 8,23)$. Podemos observar que en la metasupervisión se relevan también los aspectos que tiene que ver con el desarrollo profesional de S.

Hacia las últimas sesiones de metasupervisión comienza a aparecer la temática de los cierres en el proceso de visita domiciliaria. A diferencia de las primeras temáticas, estos contenidos no surgen como dificultad en su esencia sino más bien como un compartir con el metasupervisor el trabajo realizado. Es la metasupervisora (MS) la que activamente se detiene en estas temáticas para hacerlas parte del proceso reflexivo.

\section{Estructura de supervisión}

A través del análisis de las supervisiones podemos dar cuenta de cierta estructura dentro de cada sesión, donde, al inicio de ésta, es MS quien comienza, habitualmente, con preguntas abiertas a $S$ que presuponen una actitud de apertura y flexibilidad a temáticas y necesidades diversas de parte de esta última. Se observa en sesiones posteriores que incluso esto aparece como un acuerdo implícito, ya que $S$ inicia con un tema antes que MS le pregunte. Luego de esta pregunta inicial, aparece un tema a abordar que, en el proceso de la sesión, se reflexiona. Este proceso de reflexión se genera a partir de múltiples intervenciones de parte de MS. A esas intervenciones $S$ responde, generándose un intercambio que va enriqueciendo la mirada del problema y produciendo cambios en el modo de percibirlo y resolverlo.

Podemos desprender del análisis que MS señala como primera intervención la petición de descripción del fenómeno puesto en supervisión para luego realizar intervenciones que apuntan a ampliar la observación del tema a los distintos contextos donde está o con quienes se ve involucrado para luego favorecer en $\mathrm{S}$ la reflexión sobre su propia experiencia frente a esa problemática y traer la experiencia de los demás involucrados. Estas intervenciones van mostrando un proceso en $\mathrm{S}$ de reflexión sobre las temáticas que muestran un aumento en la empatía con las distintas personas involucradas y van generando un enriquecimiento de la mirada de $S$ sobre el fenómeno observado incluyendo cuestionamientos al modo de enfrentarlo previamente. Se ha realizado una figura desde el análisis de contenido que ofrece una mirada explícita a este proceso que se reitera a lo largo de las sesiones de supervisión.

En un momento medio de la sesión de metasupervisión ocurre una "saturación" de la reflexión (a propósito que deja de haber un diálogo o un intercambio verbal que enriquece la reflexión, con silencios, etc.), con lo que se produce un cambio de tema, y se repite el proceso descrito previamente. Hacia el final de la supervisión, habitualmente la 
metasupervisora realiza una síntesis de lo conversado en la supervisión, tomando los principales temas conversados, y en algunas oportunidades, además, refuerza positivamente los aspectos de la supervisora relacionados con la supervisión.

\section{Cambio en el paradigma de la supervisada}

Durante el análisis de las supervisiones emerge la observación de un cambio de paradigma por parte de S. Se observa un paradigma inicial sobre su rol con las monitoras y sobre su rol como supervisada. En este sentido, se plantea, en un inicio, como quien debe resolver las dificultades de las monitoras, tanto sobre su quehacer en la visita como de las necesidades diversas dentro del programa, como por ejemplo el pago de su salario a tiempo. En la relación con la supervisada, se plantea como quien trae los problemas al espacio de supervisión para recibir evaluación de su quehacer y retroalimentación sobre su alineamiento con los objetivos del programa, durante la entrevista manifiesta "... Antes de entrar a la supervisión, no tenía ninguna expectati$v a$, sino que era algo como investigación-acción, lo que si era como tener el respaldo que lo que estuviera haciendo yo estuviera alineado con lo que se espera de la investigación..." (ES, 2).

A lo largo de las supervisiones se puede observar un cambio en el paradigma respecto de su rol en su relación con las monitoras. Es así como se visualiza como quien potencia los recursos de estas últimas, validando y facilitando la participación de las monitoras en la resolución de las dificultades. Con esto, $\mathrm{S}$, pasa a tener un rol de agente de desarrollo tanto personal como profesional de sus supervisadas, lo cual genera mayor significancia y compromiso en la relación con ellas.

Por otro lado, la supervisada también va cuestionando su participación en la investigación, desde tener múltiples tareas para apoyar a las monitoras, y la gestión de su quehacer a ir delegando en otras personas del programa estos roles y funciones.

En la relación entre MS y S se observa que, a través de las supervisiones conjuntas, $\mathrm{S}$ va cambiando su modo de enfrentar la supervisión, desde una actitud más pasiva y atenta a sugerencias, a una actitud más activa y de coconstrucción de los temas a abordar y su resolución, de un estar al servicio del programa piloto a centrarse en su persona como profesional "... yo creo que eh... como que cambió ese foco hacia mi trabajo como supervisora (refiriéndose a la metasupervisión), más que así enfocada a la investigación, sino como más aterrizada, esto de la investigación era como muy sobrevuelo, digamos..." (ES, 26).

\section{Cambio en la relación}

De parte de la supervisada, inicialmente, no se observa un compromiso tan claro con el proceso de supervisión, a diferencia de MS, quien manifiesta tener un compromiso más activo y un paradigma elaborado a partir de conversaciones previas con el equipo, y donde además es parte de la producción de contenidos del programa. MS plantea en la entrevista, en relación con los objetivos que visualiza de la supervisión "... poder equilibrar entre que sea un espacio no estructurado y que ella (refiriéndose a la supervisada) pueda traer cualquier cosa, y estar como receptivo y abierta a que ella busque sus respuestas, con el poder tener un rol más activo en el sentido de estar atentos a ciertos temas que son importantes... cosa que si no sale puedo preguntar... al menos yo tengo un rol activo en el sentido de que me acuerdo de ciertos temas que estamos trabajando, entonces trato de retomarlos... trato de que se sienta cómoda, que se sienta acogida, no se sienta criticada... es súper importante que en las supervisiones la persona supervisada sienta que es un espacio pa' crecer y pa' mostrarse de verdad como está, y si uno está perdido poder decirlo, y que no sea un espacio en que uno viene a demostrar que uno lo está haciendo bien..." (EMS, 86).

Se observa, por otro lado, un cambio en la idea de $\mathrm{S}$ respecto de la utilidad de la metasupervisión para ella. En este sentido señala “... si como que... o sea desde mi perspectiva como que en un momento ya acudo a la supervision porque está establecido, en realidad por el deber, y después en realidad voy a la supervisión porque la necesito..." (ES, 48). S manifiesta como uno de los aspectos más significativos de la metasupervisión que ésta la llevara a cuestionarse sobre su quehacer como supervisada, aumentando su reflexión y, en consecuencia, generando un nuevo compromiso con su quehacer, indicando "... yo no me había cuestionado nunca las múltiples funciones que tenía, y si no fuese por ella (metasupervisora) yo nunca... yo hacía las cosas en función de mi supervisión (que ella realiza con las monitoras) y, como mi mente estaba focalizada en eso, yo no veía los medios para llegar a ella; entonces, cuando en la supervisión (refiriéndose a la metasupervisión) fuimos hablando ya de cuáles son mis múltiples roles y, sipo, y con razón estoy tan cansada, no me lo había cuestionado nunca..." (ES, 28).

Por otro lado, $S$ enriquece las posibilidades desde la metasupervisión al incluir en esta a la persona del supervisado "... si como parecido (refiriéndose a experiencias de modelos de supervisión clínica), pero se incluye también hablar de mí, también centrado no tan externalizado, sino también incorporar a la persona..." (ES, 44). 
A lo anterior se suma la percepción de cambio con relación a cómo visualiza el modelo de metasupervisión por parte de la supervisada, lo cual se puede observar a través de los cambios en las supervisiones en el tiempo, en los contenidos, en el modo de relacionarse y el modo de reflexionar sobre los casos. En este sentido, se puede percibir que la supervisada empieza a incluir contenidos en las supervisiones que van más allá de las dificultades, ocupando el espacio de metasupervisión para contar aquello en lo que le va bien, las necesidades de las monitoras, etc. como un modo de analizar las situaciones enriquecidas, y donde se incluye una toma de perspectiva de distintos actores, de niveles, de inclusión del contexto específico, e hipotetizando sobre las vivencias de cada uno de los miembros, incluyendo en esto su vivencia personal.

Se produce un traspaso de este modelo nuevo al quehacer propio de $S$ con las monitoras, en un efecto en cadena "... bueno, después me fui dando cuenta de que mi modelo de supervisión tenía mucha relación con cómo había sido supervisada yo..." (ES, 50), que, al parecer, favorece el enriquecimiento del relato de la supervisada y, por consiguiente, un mejor manejo de las dificultades, ya sea por un cambio de la mirada de la misma como por la emergencia de nuevas posibilidades de acción.

\section{Características del metasupervisor}

La metasupervisora realiza intervenciones que se visualizan como facilitadoras del proceso reflexivo de la supervisada y que favorecen el enriquecimiento de la mirada de los temas a abordar (ver figura $\mathrm{N}^{\circ} 1$ ). Se destacan aquí:

Intervenciones que apuntan a validar a la supervisada desde distintos ámbitos, utilizando refuerzo positivo, reflejo, parafraseo, validando las temáticas traídas, la propia percepción, las vivencias y los intentos de solución. Esta validación se proyecta hacia los distintos actores que van siendo puestos en escena cuando se aborda una problemática. “... encuentro impresionante el trabajo que hiciste" S5,157; "sí, y estoy pensando también cómo ha sido pa' ellas, pa' las monitoras la experiencia, qué logros han tenido, qué cosas les hubiera gustado que fueran distintas, qué expectativas se hicieron que no fue, estoy pensando en todo eso que les va a ayudar a ellas en términos de las mamás" S5,105. "Como que se nota que están súper involucradas, y eso yo creo que en gran parte es mérito tuyo, o sea no sé cómo te sentís tú, pero estaría muy satisfecha" S7,109.

A la vez, realiza intervenciones que favorecen el despliegue de información que aporta al análisis de la situación problemática, a través de preguntas abiertas (pidiendo la opinión, percepción y vivencia sobre la temática), y descripción del fenómeno relevante para la supervisada.

La metasupervisora realiza intervenciones que favorecen que la supervisada agregue información, no revelada anteriormente, a su mirada respecto de la temática, esto mediante la inclusión de distintos ni-

\section{CARACTERÍSTICAS DEL METASUPERVISOR}

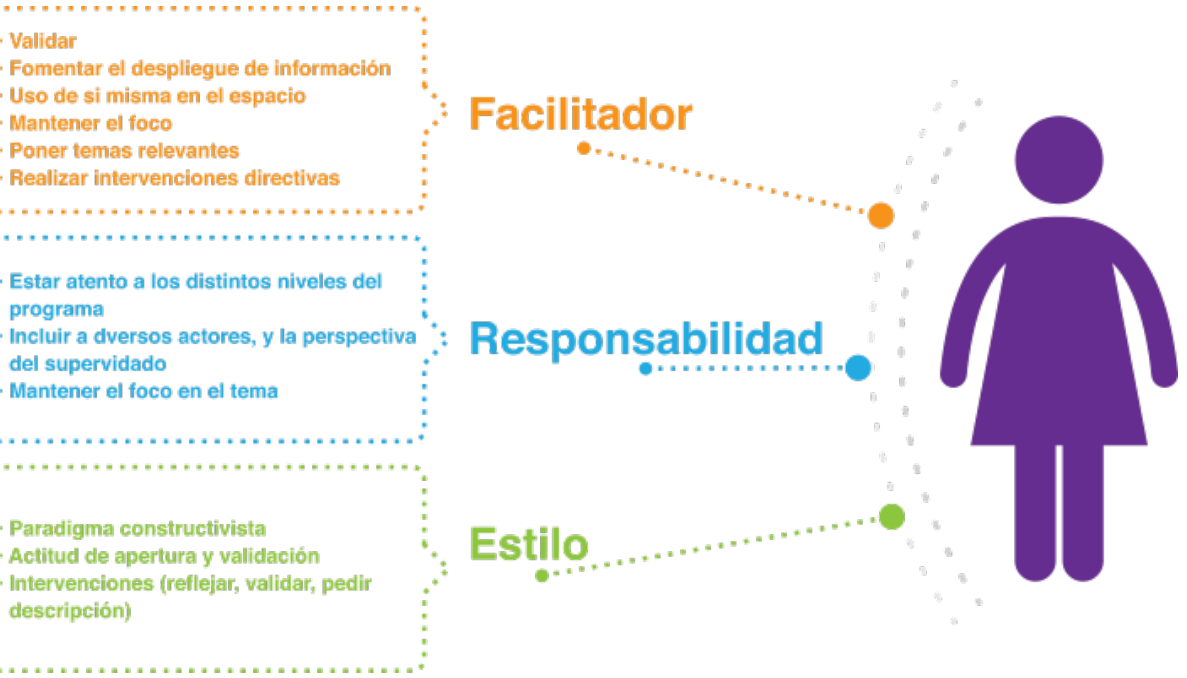


veles de contexto, ya sea incluyendo la indagación sobre los otros participantes involucrados, sus roles, sus posibles vivencias y el contexto en el que el tema está inserto, tanto a nivel físico como institucional y familiar.

El uso de sí misma, uso de experiencias previas y vivencia personal que le permiten a la supervisada mirar la situación en distintos escenarios. (Analogía entre procesos de supervisión clínico y de la monitora).

Finalmente, se realizan intervenciones tendientes a mantener el foco durante la sesión de metasupervisión de forma flexible, tanto durante la sesión de metasupervisión (explicitando los objetivos de la supervisión en algún momento) como al final de la misma, donde se realiza una síntesis sobre los distintos temas reflexionados.

Se observan otras intervenciones que no apuntan directamente a favorecer la reflexión sobre una temática traída por la supervisada, pero que dan cuenta de objetivos de la supervisión que van más allá de las necesidades de la supervisada, como poner temas que no han sido considerados por la supervisada y, en menor medida, intervenciones directivas, y sugerencias a diferentes temáticas que ya han sido reflexionadas en la supervisión.

Se resalta una constante en el accionar de la metasupervisora que apunta a favorecer el proceso reflexivo de la supervisada; además, se hace cargo de que la supervisada desarrolle un rol acorde a los objetivos del programa y acorde a las motivaciones y necesidades de la supervisada. Se observa un compromiso con la promoción de salud en los distintos niveles de los actores en la visita domiciliaria (VD), desde el bebé a la madre, la familia, las monitoras y supervisora, favoreciendo el autocuidado de cada uno de ellos. Junto con lo anterior, también se observa que la metasupervisora se compromete con las implicancias de los distintos procesos en la posible instauración futura del programa piloto a la salud pública.

Se puede desprender del análisis de las metasupervisiones y de las entrevistas una disposición de parte de la metasupervisora de mostrar apertura a través de una escucha activa, de generar un clima de confianza y dar importancia a la vivencia de la supervisada, favoreciendo el trabajo colaborativo y generando un espacio de supervisión flexible en sus temáticas. "¿Hay algo más que te preocupe de esta etapa en la que están ahora?” S7, 127.

Se percibe un compromiso de parte de la metasupervisora con su supervisada como con las demás personas involucradas (en la cadena del progra- ma de VD) a través de ofrecer ayuda, mostrarse disponible a resolver inquietudes entre sesiones de supervisión por medio de mensajes de texto o conversaciones telefónicas. Además, se visualiza un estilo reflexivo de metasupervisión que incluye la autorregulación de la propia conducta para alinearse con el modelo de supervisión elegido: en este sentido, la metasupervisora relata en la entrevista la preocupación permanente que tiene al supervisar de "poner freno a su tendencia a ser directiva".

Se explicita por parte de la metasupervisora un modelo basado en los recursos de todos los actores, validándolos y normalizando dificultades iniciales, y desde ahí generar una plataforma de seguridad, no amenazante, que permita la indagación y reflexión en el espacio de supervisión. Además, se observa un compromiso de la metasupervisora por trasladar este modo de supervisión hacia la cadena del programa, especialmente hacia la relación de la supervisora con las monitoras, con el objetivo que las monitoras puedan a su vez desarrollar una relación isomórfica con las madres. $N$ sé yo me imagino que un programa así funcionará varios años dentro de una comunidad, probablemente después la mamá que tuvo una buena experiencia le va a decir a la prima que está embarazada-oye, trata de tener la vista-, se va a armar un grupo de gente que está en torno a la maternidad y que se está apoyando, incluso a nivel comunitario uno podría ver el beneficio pá la mama, el beneficio pa' la monitora, el beneficio pa' la comunidad en que se hace...creo que esto puede tener más alcance de los que inicialmente pensamos" S7, 121.

Considerando que la supervisada ya era parte del programa piloto, y del diseño del mismo, se entiende que las expectativas sobre la supervisión y la metasupervisión no disten tanto del modelo explicitado por la metasupervisora, que incluye el centrarse en la relación más que en los contenidos, favorecer el proceso reflexivo en esa relación más que un modelo de aprendizaje a través de dar información y visualizar el proceso de supervisión como un proceso flexible en sus contenidos. Sin embargo, se observan aspectos novedosos en la mirada de la supervisada que van enriqueciendo su modelo, como son:

- Aspectos que dan cuenta de un modelo inicial de supervisión en la supervisada que dice relación con percibir un rol de compromiso con las monitoras, donde ella se muestra disponible y atenta a poder resolver todas las dudas de ellas, centrándose preferentemente en las necesidades de las monitoras y del programa. Más adelante, se percibe que la supervisada va incluyendo en 
este modelo la mirada de las propias necesidades y motivaciones para decidir sobre sus funciones y rol.

- Comprensión del efecto en cadena de los procesos de relación generados en la metasupervisión que tienen efectos en cómo ella realiza a su vez la supervisión a monitoras y cómo las monitoras se relacionan con las madres y sus familias.

\section{Resultados relacionales}

Podemos dar cuenta que se produce un proceso durante las sesiones de metasupervisión que ofrece un modo de trabajo que permite la proyección de esta instancia a otros programas de índole similar.

En este sentido emerge, en este análisis, un proceso en la interacción entre metasupervisora y supervisada (ver figura $\mathrm{N}^{\circ} 2$ ), en el cual ocurre un proceso de reflexión, que enriquece las narrativas de la supervisada, modificando el modo de comprender la dificultad puesta en el espacio de supervisión, ya que ocurriría un cambio de paradigma de la supervisada tanto de la dificultad como del lugar de los otros actores en torno a ese tema. Desde allí, se observa que la supervisada, al modificar su modo de relacionarse con los otros y de comprender el mundo de un modo distinto, traspasará este nuevo modelo a la relación con sus supervisadas, en este caso las monitoras quienes, a su vez, llevarían esta experiencia al trabajo en las visitas domiciliarias con las madres, así como a su espacio personal, ocurriendo este empoderamiento de las monitoras respecto de su quehacer. Este traspaso, y el visualizar el empoderamiento de las monitoras a través de su quehacer tanto en el ámbito de las visitas domiciliarias como en su vida personal, la llevaría a un sentido de satisfacción y desarrollo profesional de la supervisada.

Es un camino que se recorre de arriba hacia abajo (desde la metasupervisora hasta las madres), como de abajo hacia arriba, ya que el sentirse empoderadas en su relación con las madres y su quehacer en las visitas domiciliarias lleva a la supervisora a manifestar su propia satisfacción por los logros alcanzados. Se produce un cambio en la supervisada que afecta el proceso de la supervisión; el modo y los temas de la supervisión van siendo afectados desde este cambio de visión. La metasupervisora, por su parte, se muestra estable en su modo de ver y de hacer la supervisión. La supervisada se plantea en la metasupervisión, inicialmente, como en un lugar donde resolver problemas, y cumplir con los objetivos del programa para de este modo poder realizar satisfactoriamente el rol que se le ha encomendado. De este modo, aparece planteando los problemas, desde describir los hechos más que la experiencia propia asociada a esos hechos; sin embargo, se produce paulatinamente un traspaso de modelo, en que la supervisada va incluyéndose a sí en la reflexión, así como la reflexión de la participación de los otros actores involucrados.

Este cambio ocurre a partir de una postura activa de parte de la metasupervisora que se mantuvo en una actitud de receptividad, reflexión y atenta a temas que quizás no eran llevados explícitamente por la supervisada. Estos aspectos fueron permitiendo que se diera un proceso reflexivo sobre los temas abordados, que la supervisada fuera cambiando su modo de relacionarse con la metasupervisora y trasladando a su propio quehacer con las monitoras, los aspectos relacionales que se iban dando en la metasupervisión. 


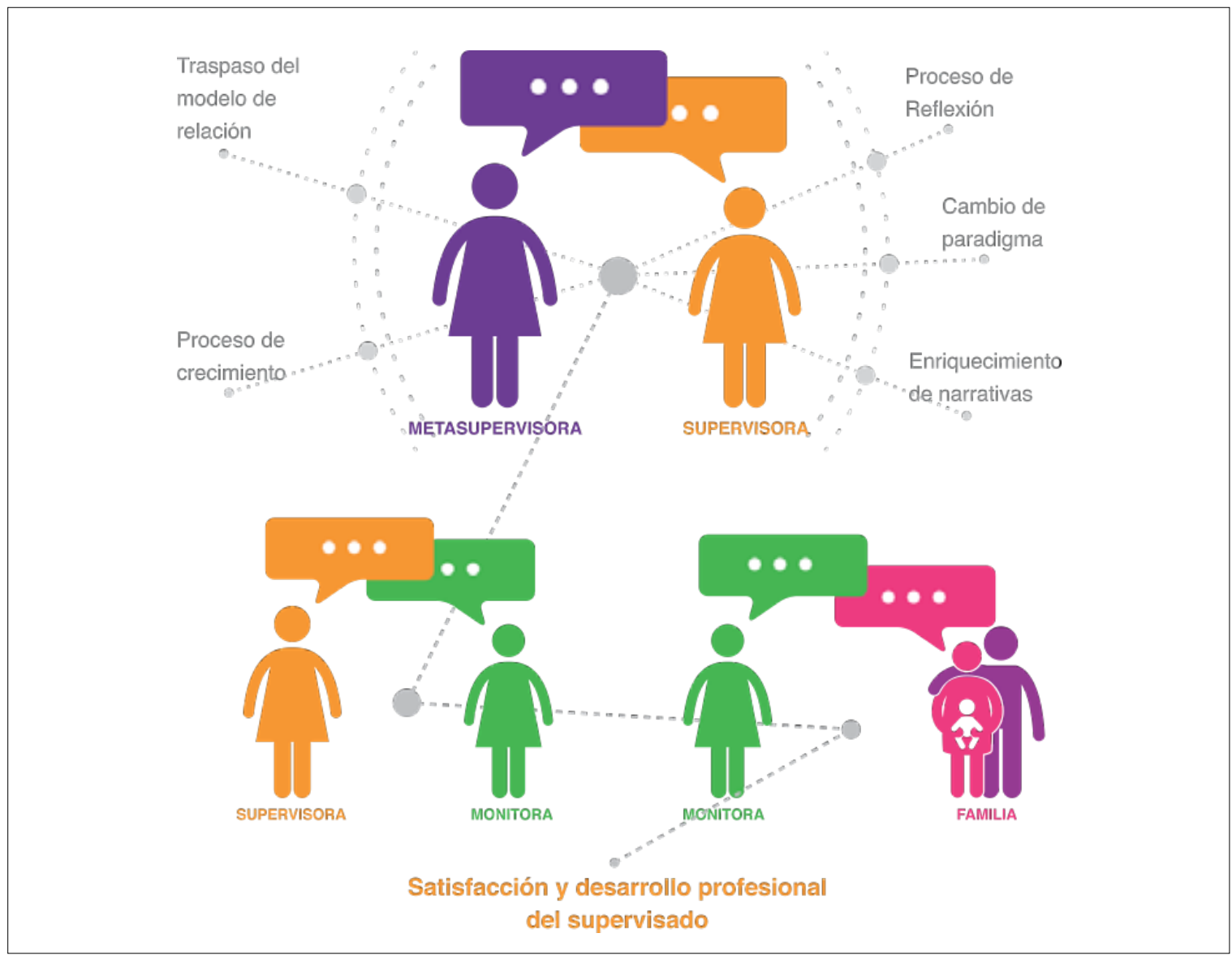

\section{Conclusión y discusiones}

Al realizar el análisis cualitativo, pudimos corroborar lo planteado por los modelos teóricos respecto de lo que ocurre en una supervisión de tipo reflexiva donde, de acuerdo a Parlakian (2001), se pueden determinar algunos elementos característicos como la reflexión, la colaboración y la regularidad.

La premisa planteada desde los estudios previos es que se produce un proceso paralelo. En la observación de la metasupervisión se pudo constatar aquello. Las relaciones se van ampliando; lo que ocurre en una relación puede inmensamente impactar otras relaciones (Geller, Whiteman y Rosenthal, 2010), donde, justamente lo que ocurre en la relación de metasupervisión, se traslada como modelo de relación a la supervisión directa a las monitoras y a la relación de éstas con las madres.

Definimos a la supervisión reflexiva como el "proceso de examinar con otra persona los pensamientos, sentimientos, acciones y reacciones evocadas en el curso del trabajo con los jóvenes y sus familias" (Eggbeer, Main y Seibel, 2007, p. 5). En este contexto, la relación de metasupervisión se da en una relación de confianza en que se habla de sus sentimientos, ideas, observaciones y los sentimientos de las visitas domiciliarias o de la relación con las monitoras y se puede observar cómo se genera ese espacio de reflexión, favorecido, entre otras variables, por el tipo de intervención y actitud definida de la metasupervisora.

Lo emergente, a partir del análisis cualitativo de la metasupervisión analizada, tuvo que ver con la generación de un contexto de satisfacción y desarrollo profesional del supervisado, donde el espacio de supervisión pasó a ser un espacio significativo para ella, aun cuando, inicialmente, sólo tenía el significado de ser parte del programa piloto para asegurar el cumplimiento de objetivos de éste.

Además, podemos señalar que se pudo vislumbrar el modo en que MS fue favoreciendo en $\mathrm{S}$ una reflexión, permitiendo este análisis dar luces de los énfasis necesarios para poder acompañar desde distintas intervenciones a otro en su proceso reflexivo sobre su trabajo de supervisión. Se puede afirmar 
que este análisis nutre el modelo de supervisión reflexiva, favoreciendo, entre otros aspectos, el poder formar a personas en este tipo de supervisión.

Igualmente, surgen aspectos sobre los cuales reflexionar con relación al modelo generado de metasupervisión.

Una cuestionamiento que surge a partir del análisis de las supervisiones es ¿cuán relevante o necesaria es la metasupervisión? De acuerdo a lo analizado, en un primer momento no aparece lo significativo del espacio, debido a que no se conocía el trabajo, lo que emergería. Sin embargo, pasado este primer momento, va instaurándose el encuentro como un lugar importante, un lugar que la supervisada visualiza como necesario y donde, desde la perspectiva de las autoras, toda la cadena de supervisión (equipo, supervisora, monitoras y madres) se ve favorecida. Sin embargo, esta necesidad puede variar, siendo quizás durante los primeros meses más perentoria para instaurar el programa efectivamente, conocer la población en que se trabaja con las madres para conocer al equipo de monitoras y el modo de relacionarse entre ellas y con las madres. Luego esta necesidad podría ir disminuyendo, pero no desaparece. En este sentido se considera que, luego de esta primera etapa, los encuentros pueden ser de modo flexible, 1 ó 2 veces al mes, según requerimiento. Dado esto, se podría pensar en instaurar la metasupervisión con una regularidad mayor en los primeros meses y con mayor distancia en los siguientes.

En esta supervisión se dio un proceso rápido de autonomía por parte de S. Se hipotetiza que la frecuencia (timing) de las supervisiones (una vez al mes) influye en la menor demanda y mayor autonomía con que $\mathrm{S}$ se mueve. Pero, por otro lado, se puede generar una dificultad ya que puede ser insuficiente el apoyo para resolver dificultades específicas. Hay que hacer el alcance que $\mathrm{S}$ está incluida en otras redes dentro del programa piloto donde también recibe retroalimentación sobre el trabajo con las monitoras y sobre las visitas domiciliarias, lo que puede mediar en neutralizar esta mayor necesidad e MS. Lo último lleva a plantear como debiese ser la frecuencia de la metasupervisión si la supervisada no es parte de otras instancias de la red, lo que podría tensionar más la necesidad de que estos encuentros fueran, incluso, de frecuencia semanal.

Se puede señalar que los desafíos para implementar de forma permanente el modelo de metasupervisión dentro de un programa de visita domiciliaria pasan por los recursos instaurados para ello, tanto de contexto como de las personas involucradas.
El presente artículo busca dar señalamientos sobre los recursos de las personas involucradas, especialmente alimentar el perfil del metasupervisor que se requiere para poder emerger un proceso reflexivo en el supervisado, como pudo verse en este estudio.

\section{Limitaciones y sugerencias}

Es posible observar que la investigación ha logrado recabar información generando una reflexión acerca de lo que ocurre en el espacio de metasupervisión. Sin embargo, se hace necesario realizar una mirada crítica al análisis realizado, dando cuenta de las limitaciones y sugerencias para estudios venideros.

En primer lugar, se puede señalar que en este estudio se da cuenta de un impacto desde la metasupervisión hacia la cadena de relaciones tanto de la supervisora con las monitoras como de la relación monitora y madre, hipotetizando que esto genera una incidencia en la relación de la madres con sus hijos. Pero este impacto no puede ser medido sólo desde la percepción de la metasupervisión, para esto deben realizarse estudios paralelos de las otras relaciones. Otra limitación metodológica es que esta investigación fue realizada en un contexto preestablecido, como lo es el proyecto de investigación sobre visitas domiciliarias, cuyas participantes analizadas (S y MS) forman parte del equipo investigativo. Por ello, ambas estaban al tanto o formaron parte de la producción de contenidos de las visitas domiciliarias y del modelo a implementar. Además, corresponde a un estudio de análisis de caso único, con las ventajas y limitantes que este tipo de estudio tiene, realizando un seguimiento a un proceso durante 8 sesiones de metasupervisión, en el que se incluyen las particularidades de cada una de las participantes, con sus visiones y recursos personales. Por ello, es posible que al trasladar este modelo de VD a la salud pública,a mayor escala, con más metasupervisoras, supervisadas, monitoras y madres a realizar seguimiento, los temas que vayan emergiendo puedan variar a partir de las necesidades específicas de cada población y región del país en particular. Esta experiencia corresponde a la primera de este tipo para cada una de las participantes, por lo que, de replicarse, ya existirá una experiencia sobre la cual basarse, sobre las expectativas del quehacer de cada una, así como de cómo resolver temas particulares. De generarse más experiencias de metasupervisión en VD, se sugiere realizar estudios sobre su impacto tanto en las madres como en las monitoras y en las supervisoras, además de evaluar su incidencia en el desarrollo y permanencias del programa dentro de los contextos donde se implemente. 


\section{Bibliografía}

CHANG, J. (2013). A contextual-functional meta-framework for counselling supervision. International Journal for the Advancement of Counselling, 35(2), 71-87. doi:http:// dx.doi.org/10.1007/s10447-012-9168-2

EGGBEER, L., MANN, T. L., \& SEIBEL, N. L. (2007). Reflective supervision: Past, present, and future. Zero to Three, 28(2), 5-9.

FLICK, U. (2004). Introducción a la investigación cualitativa. Madrid: Morata y Fundación Paideia Galiza.

FONAGY, P. \& TARGET, M. (2002). Early intervention and the development of self-regulation. En Psychoanalytic Inquiry, 22 (3), 307-335.

GLASER, B. \& STRAUSS, A. (1967). The Discovery of Grounded Theory. Chicago: Aldine Publishing Company.

GREENSPAN, S. I. (1992). Infancy and early childhood: The practice of clinical assessment and intervention with emotional and developmental challenges. Madison, CT: International Universities Press.

GELLER, J. D., FARBER, B. \& SHAFFER C. (2010). Representations of the supervisory dialogue and the development of psychotherapists. Psychotherapy: Theory, Research Practice, Training, 47 2, 210-220

GELLER, E., WHITEMAN, B., \& ROSENTHAL, H. (2010). Reflective supervision across disciplines. Zero to Three, 31(2), 31-37.

GILL, S., GREENBERG, M. T. MOON, C., \& MARGRAF, P. (2007). Home visitor competence, burnout, support and client engagement. Journal of Human Behavior in the Social Environment, 15(1), 23-44.

GUNNAR, M. R., MANGELSDORF, S., LARSON, M. \& HERTSGAARD, L. (1989). Attachment, temperament, and adrenocortical activity in infancy: A study of psychoendocrine regulation. Developmental Psychology, 25, 355-363.

HEFFRON, M.C. (2005). Reflective supervision in infant, toddler, and preschool work. In K. M. Finello (ed.), Handbook of training and practice in infant and preschool mental health. San Francisco: Jossey-Bass.

JONES HARDEN, B. (2010) Home Visitation With Psychologically Vulnerable Families Developments in the Profession and in the Professional. University of Maryland ZERO A TREE.

JONES HARDEN, B., DENMARK, N. \& SAUL, D. (2010). Understanding the needs of staff in Head Startprograms: The characteristics, perceptions, and experiences of home visitors. Children and Youth Services Review, 32(3), 371-379.

KRAEMER, S. (2006). So the cradle won't fall: Holding the staff who hold the parents in the NICU. Psychoanalytic Dialogues, 16(2), 165-179.

KRAUSE, M. \& JARAMILLO, A. (1998) Intervenciones psicológico-comunitarias en Santiago de Chile. Santiago, ed. PUC.
MORGAN, M. M. \& SPRENKLE, D. H. (2007). Toward a common-factors approach to supervision. Journal of Marital and Family Therapy, 33(1), 1-17.

PARLAKIAN, R. (2001). Observe, escuche y aprenda: La supervisión reflexiva y el trabajo basado en las relaciones. Washington, DC: CERO A TRES.

PEDERSEN, D. (1992). El dilema de lo cuantitativo y lo cualitativo de las encuestas a los métodos rápidos de investigación en salud. En F. Lolas, R. Florenzano, G. Gyarmati \& C. Trejo (Eds.), Ciencias sociales y medicina. Perspectivas latinoamericanas (pp. 201-211). Santiago: Editorial Universitaria.

RODOLFA, E., BENT, R., EISMAN, E., NELSON, P., REHM, L. RITCHIE, P. (2005). A Cube Model for Competency Development: Implications for Psychology Educators and Regulators. Professional Psychology: Research and Practice, 36, 347 - 354. doi.org/10.1037/0735-7028.36.4.347.

RUHE MARSH, L., DISSERTATION (2014) Abstracts International Section A: Humanities and Social Sciences 2 Vol 74(12-A)(E) ed. U Phoenix, US.

RUIZ, J. (1996). Metodología de la Investigación Cualitativa. Bilbao: Universidad de Deusto.

STRAUSS, A. \& CORBIN, J. (2002). Bases de la investigación cualitativa: técnicas y procedimientos para desarrollar la teoría fundamentada. Colombia: Universidad de Antioquía.

SHONKOFF Y PHILIPS (2000) Shonko, J. P. and D. Phillips (2000). From Neurons to Neighborhoods: The Science of Early Child Development. Washington, DC: National Academy Press.

TAYLOR, S. \& BODGAN, R. (1986). Introducción: Ir hacia la gente. En S. Taylor \& R. Bodgan (Eds.), Introducción a los métodos cualitativos de investigación. Buenos Aires: Paidós.

VELASCO, C., TUMA, D., ALDANA, C. (2015) Propuesta de un Modelo de Supervisión para un programa dirigido a familias de recién nacidos, en donde la Visita Domiciliaria es el eje central de la intervención. (En prensa).

WASIK, B. \& RICHARD, N. (2004). Home visitor characteristics, training, and supervision: Results of a national survey. Family Relations, 43(3), 336

WEATHERSTON, D. \& BARRON, C. (2009). What does a reflective supervisory relationship look like? In S. Scott Heller \& L. Gilkerson (Eds.). A practical guide to reflective supervision (63-82). Washington, DC: Zero to Three.

WEATHERSTON, D. J., KAPLAN-ESTRIN, M. \& GOLDBERG, S. (2009). Strengthening and recognizing knowledge, skills, and reflective practice: The Michigan association for infant mental health competency guidelines and endorsement process. Infant Mental Health Journal, 30(6), 648-663. doi:10.1002/imhj.20234.

WELD, N. (2011) A practical guide to transformative supervision for helping professions; amplifying insight. (2012). Reference and Research Book News, 27(2) Retrieved from http://ezproxy.puc.cl/docview/963529999?ac countid $=16788$ 
